\title{
Real-Time Observation of Organic Cation Reorientation in Methylammonium Lead Iodide Perovskites
}

\author{
Artem A. Bakulin, ${ }^{1,8}$ Oleg Selig, ${ }^{1}$ Huib J. Bakker, ${ }^{1}$ Yves L.A. Rezus, ${ }^{1}$ Christian \\ Müller, ${ }^{2,3,4}$ Tobias Glaser, ${ }^{3,4}$ Robert Lovrincic, ${ }^{2,3}$ Zhenhua Sun, ${ }^{5}$ Zhuoying \\ Chen, ${ }^{5}$ Aron Walsh, ${ }^{6}$ Jarvist M. Frost ${ }^{6}{ }^{6}$ Thomas L. C. Jansen ${ }^{7}$ \\ ${ }^{1}$ FOM Institute AMOLF, Science Park 104, Amsterdam, 1098 XG, The Netherlands \\ ${ }^{2}$ Institute for High-Frequency Technology, TU Braunschweig, Germany \\ ${ }^{3}$ InnovationLab GmbH, Heidelberg, Germany \\ ${ }^{4}$ Kirchhoff-Institute for Physics, Heidelberg University, Germany \\ ${ }^{5}$ LPEM-UMR 8213, ESPCI-ParisTech/CNRS/Université Pierre et Marie Curie, 10 Rue Vauquelin, \\ 75005 Paris, France \\ ${ }^{6}$ Department of Chemistry, University of Bath, Bath BA2 7AY, UK \\ ${ }^{7}$ Zernike Institute for Advanced Materials, University of Groningen, The Netherlands \\ ${ }^{8}$ University of Cambridge, Cavendish Laboratory, JJ Thomson Ave, Cambridge CB3OHE, UK
}

\begin{abstract}
:
The introduction of a mobile and polarised organic moiety as a cation in three-dimensional lead-iodide perovskites brings fascinating optoelectronic properties to these materials. The extent and the timescales of the orientational mobility of the organic cation and the molecular mechanism behind its motion remain unclear, with different experimental and computational approaches providing very different qualitative and quantitative description of the molecular dynamics. Here we use ultrafast two-dimensional vibrational spectroscopy of methylammonium (MA) lead iodide, to directly resolve the rotation of the organic cations within the $\mathrm{MAPbI}_{3}$ lattice. Our results reveal two characteristic time constants of motion. Using ab-initio molecular dynamics simulations, we identify these as a fast ( $300 \mathrm{fs})$ 'wobbling-ina-cone' motion around the crystal axis, and a relatively slow ( $\sim 3 \mathrm{ps})$ jump-like reorientation of the molecular dipole with respect to the iodide lattice. The observed dynamics are essential for understanding the electronic properties of perovskite materials.
\end{abstract}

TOC figure:
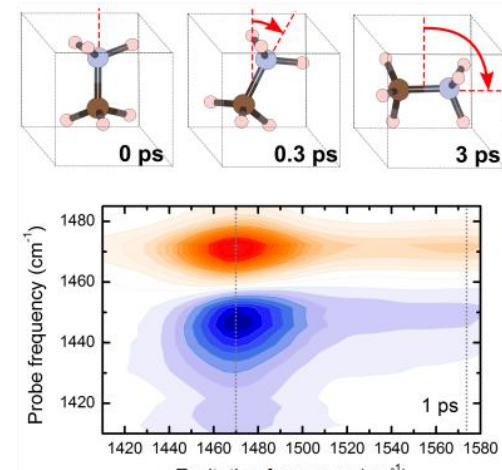

Excitation frequency $\left(\mathrm{cm}^{-1}\right)$

Keywords: methylammonium lead iodide, molecular dynamics, ultrafast spectroscopy, vibrational spectroscopy, transient anisotropy. 


\section{Main text:}

Hybrid organic-inorganic perovskites (1) are attracting a lot of attention for optoelectronic applications as they combine the solution processability of molecular electronic materials with the low exciton binding energy and high charge mobility of inorganic semiconducting crystals. (2,3) Many of these features can be related to the high dielectric constant of the material. The recent breakthroughs achieved by the application of methylammonium $\left(\mathrm{CH}_{3} \mathrm{NH}_{3}{ }^{+}\right.$or MA) lead halides (4) in photovoltaic (5-8) and light-emitting (9) devices have triggered extensive research efforts to unravel the fundamental photophysics of these material systems.

The organic cations, like MA, are not directly involved in the makeup of the valence or conduction bands. Yet they are shown to have a strong influence on the optoelectronic properties of the perovskites. $(10,11)$ The size and shape of the molecular cations, as well as their embedding in the lattice, determine the macroscopic and molecular-scale perovskite crystal structure $(10,12,13)$, which in turn determines the band gap $(11,14)$ of the material via the deformation potential. This offers the opportunity to tune the material properties through modification of the organic cation.(11) Furthermore, some of the unique properties of hybrid perovskites may be associated with the asymmetry and preferential alignment or to the dynamics of the embedded molecules. In contrast to spherically symmetric inorganic (atomic) cations, organic ions have a less symmetric shape and can hold a permanent dipole moment.(11,15) As a result, the positioning, orientation and rotational dynamics (16) of the organic moiety within the inorganic lattice can have a strong influence on the electronic system.(17) The reorientation of the organic cation with its associated dipole moment contributes to the dielectric response, and applies a crystal field to the material; both will affect the photovoltaic action. However, recent results suggest that though the choice of organic 
moieties is critical for material performance, $(18,19)$ cations do not dominate solar cell hysteresis (20) and are not essential in obtaining high open circuit voltages.(21)

A number of optoelectronic phenomena associated with molecular cation motion have been proposed based on electronic structure calculations. Cations were proposed to be responsible for the variations in dielectric constants, para- and ferroelectric behaviour,(15,22,23) current-voltage hysteresis,(10,24-26) a decrease in exciton binding energy, charge carrier segregation in the material, and dynamic disorder in valence and conduction band energies. $(27,28)$ Most of these effects are expected to be extremely sensitive to the interplay between cation-cation and cation-anion interactions, the orientational mobility of organic molecules and the time scales of the structural dynamics. The rotational mobility of organic cations in inorganic lattices has been studied for application-relevant lead-iodide and lead-bromide perovskites using MA as an organic cation (Figure 1a). The first estimates for MA reorientation were obtained using NMR spectroscopy, which pointed towards $0.2-0.4$ ps rotational correlation functions for the $\mathrm{C}-\mathrm{N}$ and $\mathrm{N}(\mathrm{C})-\mathrm{H}$ axes,(29) comparable to the time scale of the reorientation of similarly sized molecules in free space. Dielectric relaxation and calorimetry measurements confirmed the fast rotation and orientation disorder of MA in perovskite materials at room temperature.(30,31) However, some recent theoretical $(32,33)(34)$ and experimental $(22,25,35-37)$ present arguments towards longer reorientation timescales of 5-14 ps and more. Clearly, at present there is insufficient understanding of hybrid perovskite structural dynamics. What is needed is a comprehensive and direct experimental-theoretical approach that would allow one to identify the mechanistic picture behind the different timescales of the atomic and molecular motions.

In this letter, we report on an ultrafast polarization-resolved two-dimensional infrared (2DIR) vibrational spectroscopy and ab-initio molecular dynamics (MD) study of the MA cation rotation within the lattice for a set of $\mathrm{MAPbI}_{3}$ materials. Using the $\mathrm{N}^{+} \mathrm{H}_{3}$ symmetric 
bending vibration as a probe, we observe two characteristic timescales of molecular motion, which we identify as a fast ( $\sim 0.3$ ps $)$ wobbling-in-a-cone around the preferential MA dipole directions parallel to the lattice axes and relatively slow ( $>2 \mathrm{ps}$ ) jump-like reorientation of the molecular dipole (aligned with the $\mathrm{CN}$ axis) with respect to the crystal lattice. This result suggests that in $\mathrm{MAPbI}_{3}$ at room temperature the alignment of cation dipoles, if present, is likely to be of intermediate-range order.

To tackle the potential effect of crystal defects and grain boundaries on cation dynamics, we investigated $\mathrm{MAPbI}_{3}$ films made with three different techniques: (i) multilayer spin-coating, (ii) a solvent-free two-step formation from $\mathrm{PbI}_{2}$ and methylammonium-iodide (MAI) precursors, (38) and (iii) co-evaporation from $\mathrm{PbCl}_{2}$ and MAI.(39,40) For the details of film preparation see Supplementary Information. All films were deposited onto infrared (IR) transparent $\mathrm{CaF}_{2}$ substrates and had a thickness of $\sim 1 \mu \mathrm{m}$.

To observe the dynamics of molecular reorientation we used ultrafast vibrational spectroscopy, with the symmetric $\mathrm{N}^{+} \mathrm{H}_{3}$ bending vibration as a probe. Figures $1 \mathrm{~b}$-d show the structure of the MA molecule and its contribution to the perovskite IR absorption spectrum in the vibrational fingerprint region.(41) The spectrum is dominated by the $\mathrm{N}^{+} \mathrm{H}_{3}$ stretching and bending modes in the regions of $\sim 3200 \mathrm{~cm}^{-1}$ and $\sim 1500 \mathrm{~cm}^{-1}$, respectively. We attribute the weak narrow peaks in the regions around $2800 \mathrm{~cm}^{-1}$ and $1420 \mathrm{~cm}^{-1}$ to $\mathrm{CH}_{3}$ stretching and bending vibrations. These peaks are much less pronounced than the $\mathrm{N}^{+} \mathrm{H}_{3}$ vibrations due to the relatively small transition dipole moments of the $\mathrm{CH}$ vibrations as compared to the $\mathrm{NH}$ vibrations, and will not be discussed in this study. Due to the degeneracy of the three $\mathrm{NH}$ oscillators, both stretching and bending vibrations split into one symmetric and two asymmetric normal modes with orthogonal transition dipole moments. The symmetric bending mode at $1470 \mathrm{~cm}^{-1}$ is well separated in frequency from the asymmetric modes (Figure 1d) at $1580 \mathrm{~cm}^{-1}$ and has its transition dipole moment aligned along the $\mathrm{C}-\mathrm{N}$ axis of the molecule. This makes it 
possible to use the transition dipole moment of the symmetric $\mathrm{N}^{+} \mathrm{H}_{3}$ bending vibration as a probe for the reorientation of the $\mathrm{C}-\mathrm{N}$ bond and, thus, of the static molecular dipole.

To measure the orientational dynamics of the symmetric $\mathrm{N}^{+} \mathrm{H}_{3}$ bending oscillator we performed polarization-resolved 2DIR experiments $(42)(43)(44,45)$ on perovskite films in the 1400-1600 $\mathrm{cm}^{-1}$ frequency range (figure 2a). Using a collinear fast-scanning Mach-Zehnder scheme,(46) molecular vibrations were first excited by an interferometric pair of strong 100 -fs IR pump pulses. After a variable delay time, the excited state population was probed by weak broadband probe pulses with the polarizations parallel and orthogonal to the pump pulse. The isotropic signal and the anisotropy of the response (47) were calculated as:

$$
\begin{aligned}
& \Delta T_{\text {Iso }}(t)=\frac{\Delta T_{\|}(t)+2 \cdot \Delta T_{\perp}(t)}{3} \\
& r(t)=\frac{\Delta T_{\|}(t)-\Delta T_{\perp}(t)}{3 \cdot \Delta T_{\text {Iso }}(t)}
\end{aligned}
$$

Here $\Delta T_{\|}(\mathrm{t})$ and $\Delta T_{\perp}(\mathrm{t})$ stand for the time-dependent relative transmission changes for the parallel and perpendicular components of the probe, respectively. For non-interacting transition dipoles in an isotropic medium, the anisotropy decays from its initial value of 0.4 to 0 , when the orientations of the transition dipoles are completely scrambled as a result of orientation diffusion. In the isotropic signal, the effect of the reorientation is completely removed, thereby only representing population relaxation. By scanning the delay between the two pulses of the interferometric pump we obtain a frequency resolution of $\sim 5 \mathrm{~cm}^{-1}$.

Figure $2 \mathrm{~b}, \mathrm{c}$ shows the isotropic transient absorption for a co-evaporated $\mathrm{MAPbI}_{3}$ film. In this measurement, only the isotropic component was extracted. At positive delay times, the bleaching/stimulated emission of the $0-1 \mathrm{~N}^{+} \mathrm{H}_{3}$ symmetric bending transition is clearly 
observed at $1470 \mathrm{~cm}^{-1}$. The excited state 1-2 absorption from the first to the second excited state of the vibrational oscillator is observed at $1445 \mathrm{~cm}^{-1}$ and is anharmonically shifted from the $0-1$ transition by $\sim 25 \mathrm{~cm}^{-1}$. The excited-state population decays on an timescale of $\sim 3 \mathrm{ps}$ which should provide an observation window of up to 6 ps for the orientational dynamics. However, at long times we observe additional signals at $1475 \mathrm{~cm}^{-1}$ and $1420 \mathrm{~cm}^{-1}$, which do not change significantly with time. These features coincide with the linear absorption peaks (Figure 1d) and live for the times longer than 50ps, which make us to assign them to the thermal response.(47) The relaxation of high-frequency bending vibrations creates multiple lowfrequency excitations (a so-called hot ground state), which lead to the change in the absorption of the bending modes. The spectral overlap of the bleaching of the $0->1$ transition and the thermal response complicates the anisotropy analysis in this energy range. Therefore, for later anisotropy analysis, we will only consider the data from the ESA probe region $\sim 1445 \mathrm{~cm}^{-1}$, where the thermal signal is much smaller than population signal in the time window $<20 \mathrm{ps}$, as can be seen from the spectra at long delay times. With this in mind, we will use the 2D-IR spectra to (i) decouple the 'clean' rotation-related anisotropy dynamics from the thermal response and (ii) to separate the responses and anisotropy dynamics coming from the symmetric and asymmetric modes.

Figure $3 \mathrm{a}$ shows the 2D-IR spectrum of a co-evaporated $\mathrm{MAPbI}_{3}$ film at a delay of $1 \mathrm{ps}$. We use the convention of displaying the excitation frequency, $\omega_{\mathrm{ex}}$, along the horizontal axis and the probe frequency, $\omega_{\mathrm{pr}}$, along the vertical axis. As expected from the absorption spectra and the pump-probe measurements presented above, the signal associated with the bleaching of the $0-1$ transition of the symmetric bending mode is located at $\left(\omega_{\mathrm{ex}} ; \omega_{\mathrm{pr}}\right)=(1470 ; 1470) \mathrm{cm}^{-1}$ and the ESA around $(1470 ; 1445) \mathrm{cm}^{-1}$. As can be seen from the cross sections of the response for symmetric mode excitation at $\omega_{\mathrm{ex}}=1470 \mathrm{~cm}^{-1}$ (fig. $3 \mathrm{~b}$ ), at delay times longer than $10 \mathrm{ps}$, the signals associated with the excitation of the $v=1$ state are substantially influenced and then 
overtaken by the thermal response. In addition to the symmetric bending responses, the $2 \mathrm{D}$-IR spectra reveal cross peaks corresponding to the population of the symmetric mode $\omega_{\mathrm{pr}}=1470$ $\mathrm{cm}^{-1}$, following excitation of asymmetric vibrations $\omega_{\mathrm{ex}}=1560 \mathrm{~cm}^{-1}$. Due to the orthogonal orientation of the transition dipoles the response at cross peaks is strongly depolarised.

To extract the anisotropy decay originating from the symmetric mode reorientation, we focus on the responses in the center of the ESA feature at $(1470 ; 1445) \mathrm{cm}^{-1}$. Figure $3 \mathrm{c}$ presents the population dynamics measured with different pump and probe polarization and the blue dots figure $3 \mathrm{~d}$ show the extracted transient anisotropy decay for the co-evaporated $\mathrm{MAPbI}_{3}$ film. After photoexcitation, the initial anisotropy is close to 0.4 , which is the expected value for a random distribution of uncoupled dipoles.(48) The anisotropy decays on two clearly different timescales. The faster decay occurs on a timescale of $<300 \mathrm{fs}$ and decreases the anisotropy approximately by half. The longer $\sim 3$ ps decay brings the anisotropy close to zero within the 6 ps observation window. We did not analyze the dynamics at longer times because at longer delays the signal becomes dominated by the thermal response. We also note that, as the anisotropy is analyzed in the center of the ESA peak, it reflects the reorientation for the majority of the molecules. However, some minor sub-ensemble of MA ions may have a shifted IR absorption line or/and faster spectral diffusion with a more pronounced orientational dynamics. Around $500 \mathrm{fs}$, the residual anisotropy still has a relatively high value of $\sim 0.2$ for all samples. While the fast time-scale observed here agrees with the $400 \mathrm{fs}$ timescale reported in previous NMR experiments, (29) the slow timescale of 3 ps has not been observed before. Longer ( $\sim 5 \mathrm{ps})$ timescales were predicted based on selective dynamics (artificially constrained frozen lead positions) MD simulations (14) and measured (5-14 ps) with quasi elastic neutron scattering on bulk material.(37,49) Independent of the preparation protocol and film morphology, all our thin-film samples demonstrate similar anisotropy dynamics (figure 4a). We can hence exclude an influence of solvent residuals on the observed time scales, as two of 
the samples were fabricated with solvent free methods. We speculate that this explains part of the discrepancy between the reorientation times presented here and those recently derived from neutron scattering.(37)

To obtain molecular-scale mechanistic insight into the origin and details of organic cation dynamics we undertake ab-initio molecular dynamic (MD) simulations starting in a pseudo-cubic geometry and at temperatures of 150,300 and $450 \mathrm{~K}$. To validate the simulations and to allow for a direct comparison to experimental results, the MD trajectories are used directly to calculate the absorption and the full 2D-IR response of the $\mathrm{MA} \mathrm{N} \mathrm{N}^{+} \mathrm{H}_{3}$ bending vibrations. (50) (51)

Figure 1d shows that the $\mathrm{N}^{+} \mathrm{H}_{3}$ bending absorption spectrum calculated with the procedure described above reproduces the experimental results well. The additional small peaks observed in the experimental data are probably associated with the $\mathrm{CH}_{3}$ bending modes; the slightly different absorption cross section ratio for the symmetric and asymmetric modes is likely to originate from our assumption that the transition dipole direction of the local $\mathrm{NH}$ bendings mode are perpendicular to the NH bonds. Figure $4 \mathrm{~b}$ shows the calculated $2 \mathrm{D}$-IR spectrum (perpendicular polarisations), which also reproduces the experimental results (Figure 3). This proves the adequacy of the applied method for calculating the spectroscopic observables and, in turn, allows the further analysis of anisotropy and orientation dynamics as taken explicitly from the MD simulations.

The red line in Figure $3 d$ shows the anisotropy decay in the $(1470 ; 1445) \mathrm{cm}^{-1}$ region of the calculated 2D-IR spectra, i.e. in the same area used to extract the experimental anisotropies. The simulated curve matches the experimental dynamics very well. Only the short-time component of the decay is slightly faster, which is likely to be the effect of experimental time resolution. First of all, this confirms the validity of the MD simulations and 
calculated orientation dynamics. Secondly, the theory-experiment match indicates the absence of intermolecular hopping of vibrational excitations. Intermolecular energy transfer is not included in the theoretical calculations and, in case it would play a role, the experimental anisotropy should decay much faster as seen in other materials with fast vibrational excitation transfer.(47,52) Finally, the validated MD calculations can be used to identify the molecular picture behind the two observed timescales in anisotropy dynamics.

We first verify that the anisotropy decay is due to rotation and not intramolecular vibrational population transfer. Simulating the 2D-IR spectra and extracting the anisotropy decay with fixed molecular orientations gives an anisotropy of 0.4 for all waiting times demonstrating that the origin of the observed decay is solely due to reorientation of the MA ions. This is not the case for the asymmetric peak at $1570 \mathrm{~cm}^{-1}$, where two degenerate modes rapidly mix. We also calculated $2 \mathrm{D}$-IR spectra at $150 \mathrm{~K}$ and $450 \mathrm{~K}$ and the extracted transient anisotropies are shown in Figure 3d. It is apparent that at $150 \mathrm{~K}$ the anisotropy does not decay below 0.3 , and that the anisotropy dynamics at $450 \mathrm{~K}$ are faster and lead to a nearly complete decay at 6 ps. Previous studies indicated a preferential orientation of the MD dipole parallel to the lattice axes (33) suggesting that at low temperatures the molecular dipole axis may be pinned to the lattice axes (fig. 4c, left). In Figure 4d histograms of the distribution of the angle between the molecular axis and the nearest of the lattice axis, which are equivalent in the simulated pseudo-cubic symmetry, are illustrated for the three simulated temperatures normalized to a random isotropic distribution of dipole directions. For 150 and $300 \mathrm{~K}$ there is a larger probability of finding an angle below 30 degree and lower probability of finding a larger angle. The $450 \mathrm{~K}$ data hardly deviate from the isotropic distribution. This suggests that the MA dipole preferentially points along one of the three lattice axes and is at $300 \mathrm{~K}$ confined within a semi-cone angle of about 30 degrees from each of these axes. We can consider a 
wobbling-in-a-cone/angular jump model,(53) where the rotational anisotropy is given by the equation

$$
r(t)=\frac{2}{5}\left(S^{2} \exp \left(-\frac{t}{\tau_{\text {jump }}}\right)+\left(1-S^{2}\right) \exp \left(-\left(\frac{1}{\tau_{\text {jump }}}+\frac{1}{\tau_{\text {wob }}}\right) t\right)\right)
$$

and where $\mathrm{r}(\mathrm{t})$ is the anisotropy, $\tau_{\text {jump }}$ is the effective jump time scale for 90 degree jumps, $\tau_{w o b}$ is the time scale of the wobbling motion, and $\mathrm{S}$ is a constant depending on the semi-cone angle, $\Theta_{C}$, of the wobbling motion given by the equation

$$
S=\cos \left(\Theta_{C}\right)\left(1+\cos \left(\Theta_{C}\right)\right) / 2
$$

A biexponential fit to the simulated data results in a semi-cone angle of 31 degrees $(S=0.625)$ from both the $150 \mathrm{~K}$ and $300 \mathrm{~K}$ data. The wobbling-in-a-cone timescale slows down from 130 fs to $1.1 \mathrm{ps}$, when cooling from $300 \mathrm{~K}$ to $150 \mathrm{~K}$. We note that at $300 \mathrm{~K}$ the wobbling time fits well the $150 \mathrm{~cm}^{-1}$ vibration previously observed using Raman spectroscopy and assigned to librational motion,(54) which is the same as wobbling-in-a-cone motion. The time scale for the jump type motion is too long to fit for $150 \mathrm{~K}$ and 2.7 ps at $300 \mathrm{~K}$ (see also fig S5 in SI). The $450 \mathrm{~K}$ data can be fitted with a single time scale of $570 \mathrm{fs}$ and a small offset of 0.07 suggesting that the motion at this temperature is slowed down, but no longer confined by the barriers. The small offset hints at the presence of an optimal orientation of the cation along one of the crystal axes, but may also be due to the error bars in the simulation data as at $450 \mathrm{~K}$ the cubic crystal structure prevails. It should be noted that all simulations were done for the pseudo-cubic phase and that experimentally at low temperature the structure is orthorhombic. This phase transition is thus not accounted for in the present simulations.

The combined experimental and theoretical results demonstrate that in the studied samples at $300 \mathrm{~K}$ the MA dipoles reorient on two distinct time scales. These are interpreted as fast local wobbling-in-a-cone motion and slow 90 degrees jumps, respectively. We cannot fully 
exclude a slower decay component, but the upper limit for the contribution of such a component is in the order of 0.07 , corresponding to $<20 \%$ of all MA molecules moving on a slower time scale. These measurements thus dismisses the existence of large, slowly interconverting ferroelectric domains. However, we cannot rule out that the jump motion involves concerted motion of correlated MA domains.

To conclude, ultrafast 2D-IR vibrational anisotropy spectroscopy on the $\mathrm{N}^{+} \mathrm{H}_{3}$ symmetric bending vibration of MA and ab-initio MD were used to resolve in time the methylammonium cation rotation in different $\mathrm{MAPbI}_{3}$ perovskites films. Excellent agreement was found between the experiment and the simulations. For all materials we observed similar dynamics with two characteristic timescales of molecular motion, which we identified as a fast wobbling-in-a-cone motion around the lattice axes and a relatively slow ( $\sim 3$ ps) jump-like reorientation of the molecular dipole (aligned with $\mathrm{CN}$ axis) between the different lattice axes. These timescales are directly relatable to the contribution to the dielectric response that comes from the molecular motion. Such a contribution is of extreme importance for the device physics of these materials, as the stability of excitons, polarons, and other quasi-particle states will depend on the response time of the dielectric constant. These findings represent the first realtime measurement of organic cation motion in organic-inorganic perovskite materials and provide access to the molecular mechanisms behind the optoelectronic properties of these materials.

\section{Acknowledgements:}

We thank Maxim Pschenichnikov for useful discussions. This work was supported by the Netherlands Organization for Scientific Research (NWO) through the "Stichting voor Fundamenteel Onderzoek der Materie" (FOM) research program. A.A.B. also acknowledges a VENI grant from the NWO. A.A.B. is currently a Royal Society University Research Fellow. 
Z.S. and Z.C. acknowledge the ANR-2011-JS09-004-01-PvCoNano project and the EU Marie Curie Career Integration Grant (303824). A.A.B., Z.S. and Z.C. thank Dutch-French Academy for the support through van Gogh grant.

Supporting Information Available: sample preparation and morphology characterization, ultrafast spectroscopy setup description, additional transient absorption and 2DIR spectra, details of molecular dynamics simulations and 2D IR spectra calculations, modelled cation orientation trajectories. This material is available free of charge via the Internet http://pubs.acs.org . 


\section{Figures:}
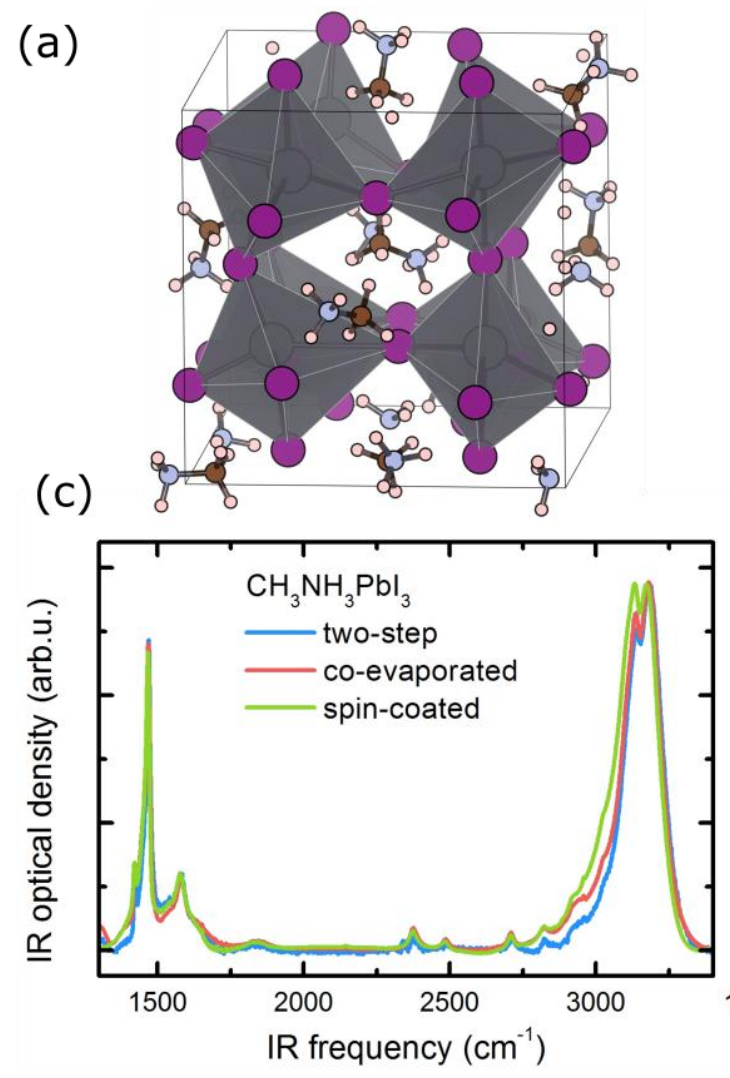

(b)

(d)
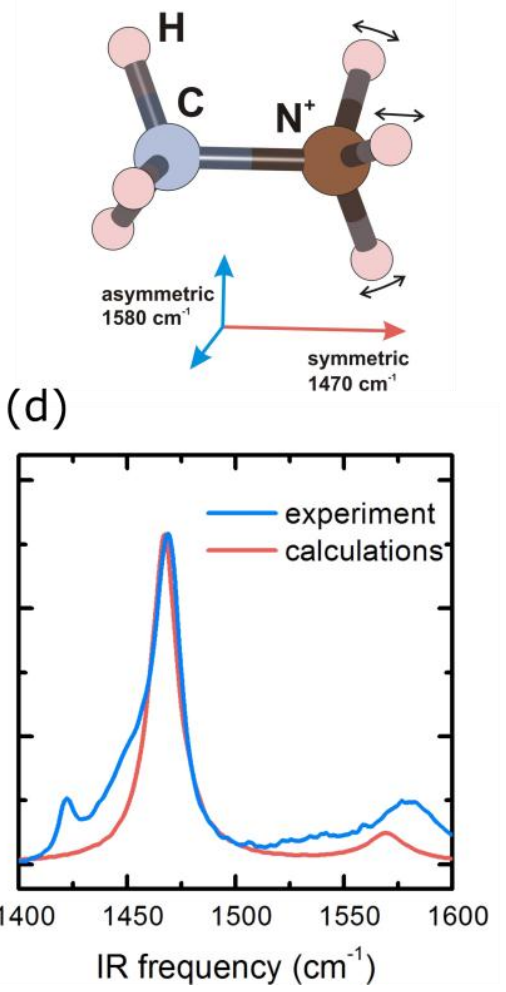

Figure 1. Perovskite material under study: (a) A snapshot from the molecular dynamics simulation showing the structure of the $\mathrm{MAPbI}_{3}$ perovskite. (b) The MA molecule and the orientation of the transition dipoles of the $\mathrm{NH}_{3}$ bending vibrations. (c) IR absorption spectra of $\mathrm{MAPbI}_{3}$ films prepared using different methods. (d) Absorption spectra of a $\mathrm{MAPbI}_{3}$ film in the $\mathrm{NH} / \mathrm{CH}$ bending vibrational region. The red line shows the calculated absorption spectrum of the $\mathrm{NH}_{3}$ bending modes (on the basis of the MD simulation). 
(a)

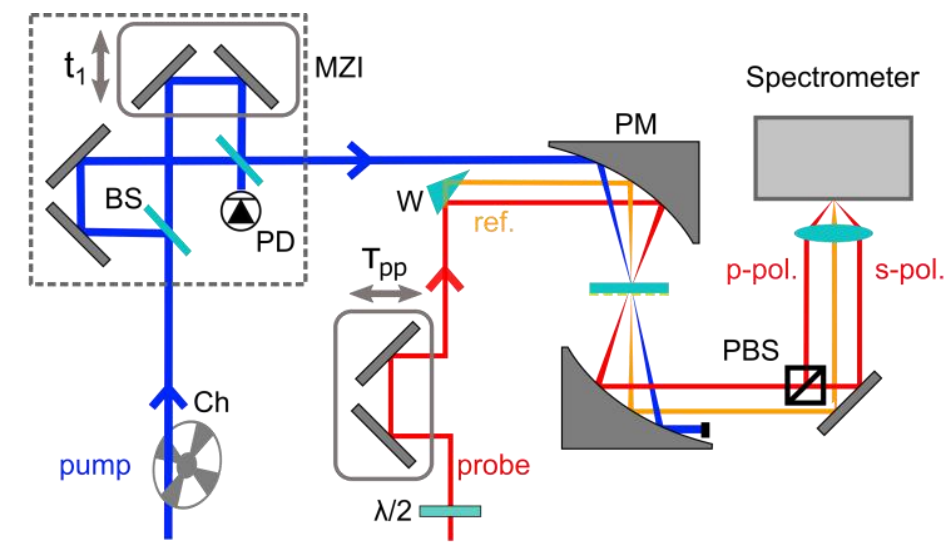

(b)

(c)
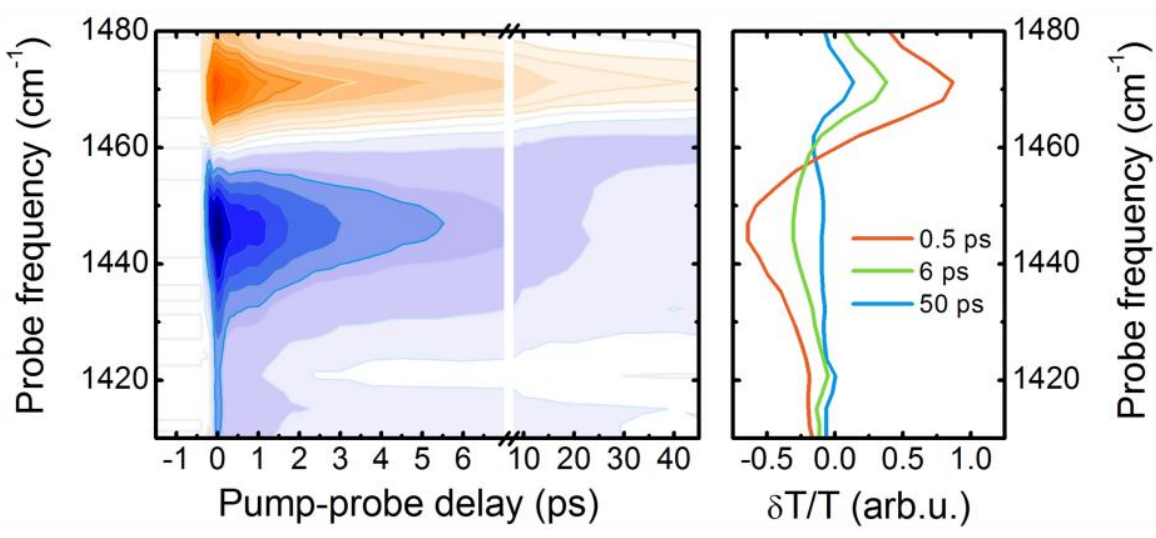

Figure 2. Vibrational 2D spectroscopy: (a) Experimental setup for the 2D-IR polarisation resolved measurements. (b) Isotropic pump-probe transient spectra of a co-evaporated perovskite film measured using broadband excitation. (c) Isotropic pump-probe responses at different pump-probe delay times. 


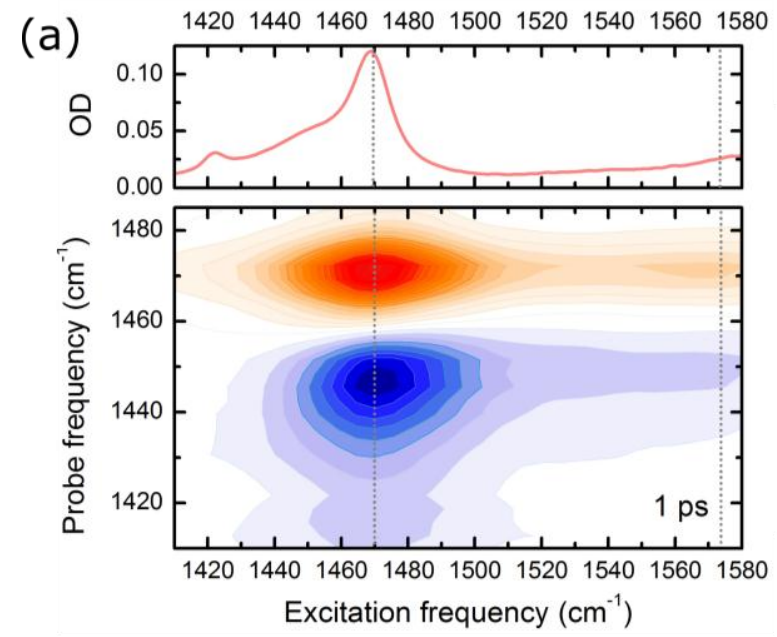

(c)

(b)
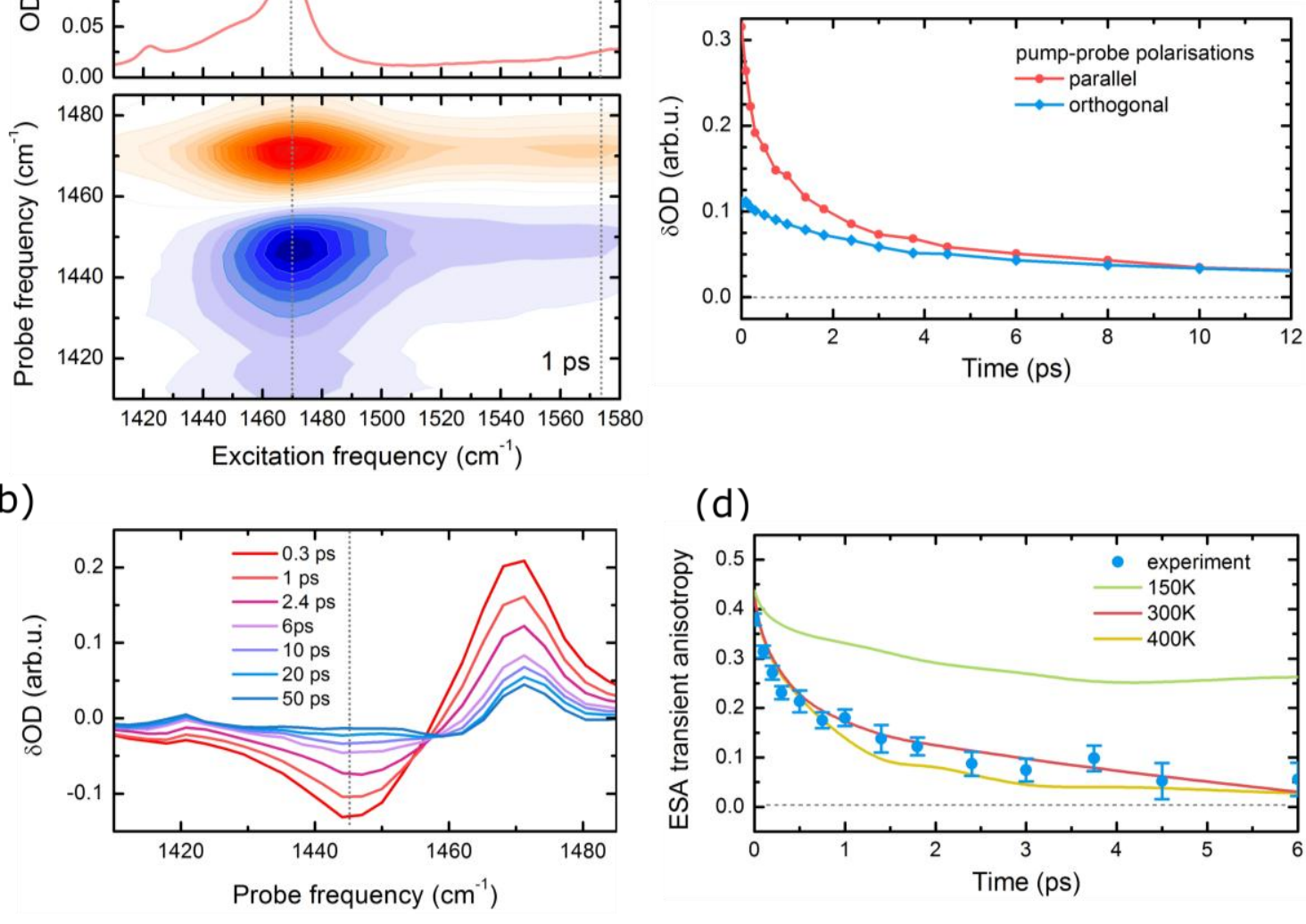

(d)

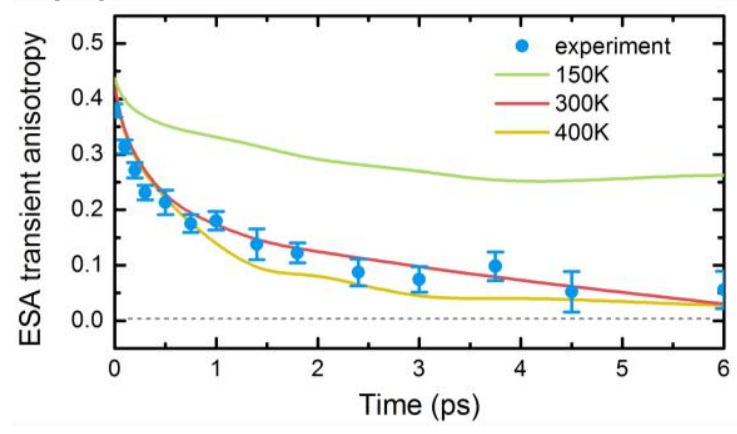

Figure 3. 2DIR response of NH bending vibrations: (a) Isotropic 2D spectra of coevaporated perovskite film (average over three samples) 1 ps delay time. The different colours correspond to 5\% steps from the maximum amplitude. Upper panel shows the corresponding absorption spectrum. (b) Cross sections through the 2D spectrum of the co-evaporated perovskite at $\omega_{e x}=1470 \mathrm{~cm}^{-1}$ for different delay times. (c) The dynamics of the excited-state absorption in the $\left(\omega_{e x}=1470 \mathrm{~cm}^{-1} ; \omega_{p r}=1445 \mathrm{~cm}^{-1}\right)$ point of 2D spectrum measured for parallel and orthogonal pump and probe pulses polarisations. (d) Anisotropy dynamics associated with the data in panel (c) with $95 \%$ confidence interval indicated. The solid lines represent the transient anisotropy dynamics calculated based on the MD simulations at different temperatures. 
(a)

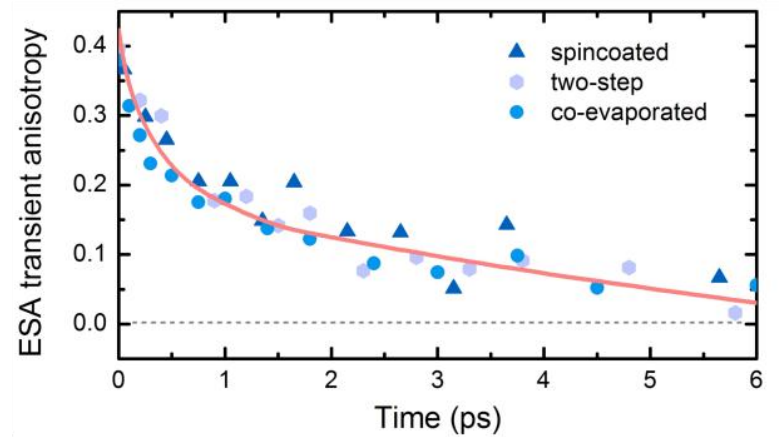

(b)

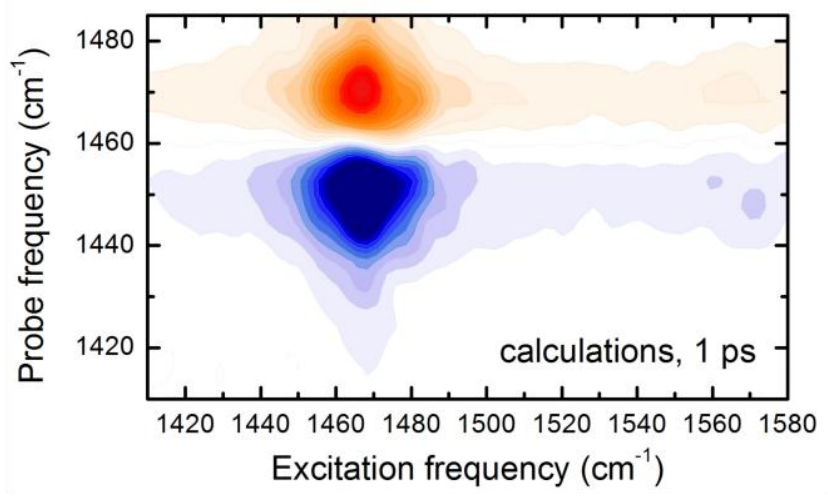

(c)

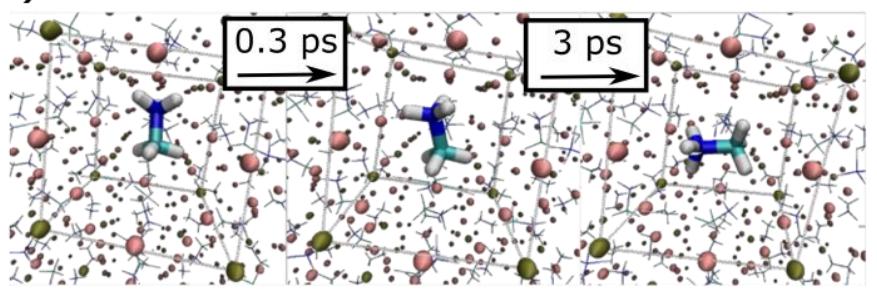

(d)

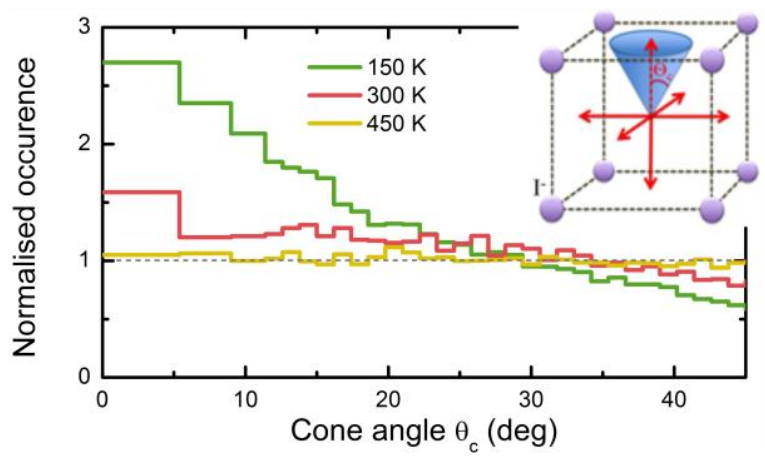

Figure 4. (a) Transient anisotropy in the peak of ESA response (excitation $=1470 \mathrm{~cm}^{-1}$; probe $=1445 \mathrm{~cm}^{-1}$ ) for samples prepared using different techniques. The red line is a guide to the eye showing the transient anisotropy dynamics calculated based on MD simulations at $300 \mathrm{~K}$. The time resolution was $\sim 100$ fs for co-evaporated film and $\sim 300$ fs for spincoated and two-step fabricated films. (b) The example of 2D-IR spectrum calculated with MD simulations. (c) An artist impression illustrating the motion of organic cation within the perovskite material. (d) Histogram of the simulated angular distribution of the MA dipoles at different temperatures normalized to the isotropic distribution. The inset illustrates the definition of the cone angle. 


\section{References:}

(1) Mitzi, D. B. Synthesis, Structure, and Properties of Organic-Inorganic Perovskites and Related Materials. In Progress in Inorganic Chemistry; John Wiley \& Sons, Inc.: 2007, p 1-121.

(2) Snaith, H. J. Perovskites: The Emergence of a New Era for Low-Cost, High-Efficiency Solar Cells. J. Phys. Chem. Lett. 2013, 4, 3623-3630.

(3) Green, M. A.; Ho-Baillie, A.; Snaith, H. J. The Emergence of Perovskite Solar Cells. Nat. Photonics 2014, 8, 506-514.

(4) Weber, D. Z., CH3NH3PbX3, a Pb(II)-System with Cubic Perovskite Structure. Z. Naturforsch. 1978, 33b, 1443-1445.

(5) Heo, J. H.; Im, S. H.; Noh, J. H.; Mandal, T. N.; Lim, C.-S.; Chang, J. A.; Lee, Y. H.; Kim, H.-j.; Sarkar, A.; NazeeruddinMd, K.et al. Efficient Inorganic-Organic Hybrid Heterojunction Solar Cells Containing Perovskite Compound and Polymeric Hole Conductors. Nat. Photonics 2013, 7, 486-491.

(6) Lee, M. M.; Teuscher, J.; Miyasaka, T.; Murakami, T. N.; Snaith, H. J. Efficient Hybrid Solar Cells Based on Meso-Superstructured Organometal Halide Perovskites. Science 2012, 338, 643647.

(7) Kim, H.-S.; Lee, C.-R.; Im, J.-H.; Lee, K.-B.; Moehl, T.; Marchioro, A.; Moon, S.-J.; Humphry-Baker, R.; Yum, J.-H.; Moser, J. E.et al. Lead lodide Perovskite Sensitized All-Solid-State Submicron Thin Film Mesoscopic Solar Cell with Efficiency Exceeding 9\%. Sci. Rep. 2012, 2, 591.

(8) Noh, J. H.; Im, S. H.; Heo, J. H.; Mandal, T. N.; Seok, S. I. Chemical Management for Colorful, Efficient, and Stable Inorganic-Organic Hybrid Nanostructured Solar Cells. Nano Letters 2013, 13, 1764-1769.

(9) Tan, Z.-K.; Moghaddam, R. S.; Lai, M. L.; Docampo, P.; Higler, R.; Deschler, F.; Price, M.; Sadhanala, A.; Pazos, L. M.; Credgington, D.et al. Bright Light-Emitting Diodes Based on Organometal Halide Perovskite. Nat. Nanotechnol. 2014, 9, 687-692.

(10) Stoumpos, C. C.; Malliakas, C. D.; Kanatzidis, M. G. Semiconducting Tin and Lead lodide Perovskites with Organic Cations: Phase Transitions, High Mobilities, and near-Infrared Photoluminescent Properties. Inorganic Chemistry 2013, 52, 9019-9038.

(11) Amat, A.; Mosconi, E.; Ronca, E.; Quarti, C.; Umari, P.; Nazeeruddin, M. K.; Grätzel, M.; De Angelis, F. Cation-Induced Band-Gap Tuning in Organohalide Perovskites: Interplay of Spin-Orbit Coupling and Octahedra Tilting. Nano Letters 2014, 14, 3608-3616.

(12) Baikie, T.; Fang, Y.; Kadro, J. M.; Schreyer, M.; Wei, F.; Mhaisalkar, S. G.; Graetzel, M.; White, T. J. Synthesis and Crystal Chemistry of the Hybrid Perovskite CH3NH3Pbl3 for Solid-State Sensitised Solar Cell Applications. J. Mat. Chem. A 2013, 1, 5628-5641.

(13) Wu, X.; Trinh, M. T.; Zhu, X. Excitonic Many-Body Interactions in Two-Dimensional Lead lodide Perovskite Quantum Wells. J. Phys. Chem. C 2015, 119, 14714-14721.

(14) Mosconi, E.; Quarti, C.; Ivanovska, T.; Ruani, G.; De Angelis, F. Structural and Electronic Properties of Organo-Halide Lead Perovskites: A Combined Ir-Spectroscopy and Ab Initio Molecular Dynamics Investigation. Phys. Chem. Chem. Phys. 2014, 16, 16137-16144.

(15) Grinberg, I.; West, D. V.; Torres, M.; Gou, G.; Stein, D. M.; Wu, L.; Chen, G.; Gallo, E. M.; Akbashev, A. R.; Davies, P. K.et al. Perovskite Oxides for Visible-Light-Absorbing Ferroelectric and Photovoltaic Materials. Nature 2013, 503, 509-512.

(16) Baikie, T.; Barrow, N. S.; Fang, Y.; Keenan, P. J.; Slater, P. R.; Piltz, R. O.; Gutmann, M.; Mhaisalkar, S. G.; White, T. J. A Combined Single Crystal Neutron/X-Ray Diffraction and Solid-State Nuclear Magnetic Resonance Study of the Hybrid Perovskites $\mathrm{CH} 3 \mathrm{NH} 3 \mathrm{PbX} 3(\mathrm{X}=\mathrm{I}, \mathrm{Br}$ and $\mathrm{Cl})$. J. Mat. Chem. A 2015, 3, 9298-9307.

(17) Ma, J.; Wang, L.-W. Nanoscale Charge Localization Induced by Random Orientations of Organic Molecules in Hybrid Perovskite CH3NH3PbI3. Nano Letters 2014, 15, 248-253.

(18) Lee, J.-W.; Seol, D.-J.; Cho, A.-N.; Park, N.-G. High-Efficiency Perovskite Solar Cells Based on the Black Polymorph of HC(NH2)2PbI3. Adv. Mater. 2014, 26, 4991-4998. 
(19) Jeon, N. J.; Noh, J. H.; Yang, W. S.; Kim, Y. C.; Ryu, S.; Seo, J.; Seok, S. I. Compositional Engineering of Perovskite Materials for High-Performance Solar Cells. Nature 2015, 517, 476-480.

(20) Beilsten-Edmands, J.; Eperon, G. E.; Johnson, R. D.; Snaith, H. J.; Radaelli, P. G. NonFerroelectric Nature of the Conductance Hysteresis in $\mathrm{CH} 3 \mathrm{NH} 3 \mathrm{~Pb} 33$ Perovskite-Based Photovoltaic Devices. Appl. Phys. Lett. 2015, 106, 173502.

(21) Kulbak, M.; Cahen, D.; Hodes, G. How Important Is the Organic Part of Lead Halide Perovskite Photovoltaic Cells? Efficient CsPbBr3 Cells. J. Phys. Chem. Lett. 2015, 6, 2452-2456.

(22) Kutes, Y.; Ye, L.; Zhou, Y.; Pang, S.; Huey, B. D.; Padture, N. P. Direct Observation of Ferroelectric Domains in Solution-Processed CH3NH3Pbl3 Perovskite Thin Films. J. Phys. Chem. Lett. 2014, 5, 3335-3339.

(23) Zheng, F.; Takenaka, H.; Wang, F.; Koocher, N. Z.; Rappe, A. M. First-Principles Calculation of the Bulk Photovoltaic Effect in $\mathrm{CH} 3 \mathrm{NH} 3 \mathrm{PbI} 3$ and $\mathrm{CH} 3 \mathrm{NH} 3 \mathrm{PbI} 3-\mathrm{XClx}$. J. Phys. Chem. Lett. 2014, 6, 31-37.

(24) Baumann, A.; Tvingstedt, K.; Heiber, M. C.; Väth, S.; Momblona, C.; Bolink, H. J.; Dyakonov, V. Persistent Photovoltage in Methylammonium Lead lodide Perovskite Solar Cells. APL Materials 2014, 2, 081501.

(25) Snaith, H. J.; Abate, A.; Ball, J. M.; Eperon, G. E.; Leijtens, T.; Noel, N. K.; Stranks, S. D.; Wang, J. T.-W.; Wojciechowski, K.; Zhang, W. Anomalous Hysteresis in Perovskite Solar Cells. J. Phys. Chem. Lett. 2014, 5, 1511-1515.

(26) Wei, J.; Zhao, Y.; Li, H.; Li, G.; Pan, J.; Xu, D.; Zhao, Q.; Yu, D. Hysteresis Analysis Based on the Ferroelectric Effect in Hybrid Perovskite Solar Cells. J. Phys. Chem. Lett. 2014, 5, 3937-3945.

(27) Quarti, C.; Mosconi, E.; De Angelis, F. Interplay of Orientational Order and Electronic Structure in Methylammonium Lead lodide: Implications for Solar Cell Operation. Chem, Mater. 2014, 26, 6557-6569.

(28) Yin, W.-J.; Yang, J.-H.; Kang, J.; Yan, Y.; Wei, S.-H. Halide Perovskite Materials for Solar Cells: A Theoretical Review. J. Mater. Chem. A 2015, 3, 8926-8942.

(29) Wasylishen, R. E.; Knop, O.; Macdonald, J. B. Cation Rotation in Methylammonium Lead Halides. Solid State Comm. 1985, 56, 581-582.

(30) Onoda-Yamamuro, N.; Matsuo, T.; Suga, H. Calorimetric and Ir Spectroscopic Studies of Phase Transitions in Methylammonium Trihalogenoplumbates (li)†. J. Phys. Chem. Solids 1990, 51, 1383-1395.

(31) Onoda-Yamamuro, N.; Matsuo, T.; Suga, H. Dielectric Study of $\mathrm{CH} 3 \mathrm{NH} 3 \mathrm{PbX} 3(\mathrm{X}=\mathrm{Cl}$, $\mathrm{Br}$, I). J. Phys. Chem. Solids 1992, 53, 935-939.

(32) Frost, J. M.; Butler, K. T.; Brivio, F.; Hendon, C. H.; van Schilfgaarde, M.; Walsh, A. Atomistic Origins of High-Performance in Hybrid Halide Perovskite Solar Cells. Nano Letters 2014, 14, 2584-2590.

(33) Frost, J. M.; Butler, K. T.; Walsh, A. Molecular Ferroelectric Contributions to Anomalous Hysteresis in Hybrid Perovskite Solar Cells. APL Materials 2014, 2, 081506.

(34) Carignano, M. A.; Kachmar, A.; Hutter, J. Thermal Effects on Ch3nh3pbi3 Perovskite from Ab Initio Molecular Dynamics Simulations. J. Phys. Chem. C 2015, 119, 8991-8997.

(35) Chi, L.; Swainson, I.; Cranswick, L.; Her, J.-H.; Stephens, P.; Knop, O. The Ordered Phase of Methylammonium Lead Chloride CH3NH3Pbl3. J. Solid State Chem. 2005, 178, 1376-1385.

(36) Fan, Z.; Xiao, J.; Sun, K.; Chen, L.; Hu, Y.; Ouyang, J.; Ong, K. P.; Zeng, K.; Wang, J. Ferroelectricity of CH3NH3PbI3 Perovskite. J. Phys. Chem. Lett. 2015, 1155-1161.

(37) Leguy, A. M. A.; Frost, J. M.; McMahon, A. P.; Sakai, V. G.; Kochelmann, W.; Law, C.; Li, X.; Foglia, F.; Walsh, A.; O/'Regan, B. C.et al. The Dynamics of Methylammonium Ions in Hybrid Organic-Inorganic Perovskite Solar Cells. Nat. Commun. 2015, 6, 7124.

(38) Hao, F.; Stoumpos, C. C.; Liu, Z.; Chang, R. P. H.; Kanatzidis, M. G. Controllable Perovskite Crystallization at a Gas-Solid Interface for Hole Conductor-Free Solar Cells with Steady Power Conversion Efficiency over 10\%. J. Am. Chem. Soc. 2014, 136, 16411-16419. 
(39) Polander, L. E.; Pahner, P.; Schwarze, M.; Saalfrank, M.; Koerner, C.; Leo, K. HoleTransport Material Variation in Fully Vacuum Deposited Perovskite Solar Cells. APL Materials 2014, 2, 081503.

(40) Liu, M.; Johnston, M. B.; Snaith, H. J. Efficient Planar Heterojunction Perovskite Solar Cells by Vapour Deposition. Nature 2013, 501, 395-398.

(41) Glaser, T.; Müller, C.; Sendner, M.; Krekeler, C.; Semonin, O. E.; Hull, T. D.; Yaffe, O.; Owen, J. S.; Kowalsky, W.; Pucci, A.et al. Infrared Spectroscopic Study of Vibrational Modes in Methylammonium Lead Halide Perovskites. J. Phys. Chem. Lett. 2015, 6, 2913-2918.

(42) Hamm, P.; Lim, M. H.; Hochstrasser, R. M. Structure of the Amide I Band of Peptides Measured by Femtosecond Nonlinear-Infrared Spectroscopy. J. Phys. Chem. B 1998, 102, 6123-6138.

(43) Zheng, J.; Kwak, K.; Fayer, M. D. Ultrafast 2D IR Vibrational Echo Spectroscopy. Acc. Chem. Res. 2007, 40, 75-83.

(44) Skoff, D. R.; Laaser, J. E.; Mukherjee, S. S.; Middleton, C. T.; Zanni, M. T. Simplified and Economical 2D IR Spectrometer Design Using a Dual Acousto-Optic Modulator. Chem. Phys. 2013, 422, 8-15.

(45) Ramasesha, K.; Roberts, S. T.; Nicodemus, R. A.; Mandal, A.; Tokmakoff, A. Ultrafast 2D IR Anisotropy of Water Reveals Reorientation During Hydrogen-Bond Switching. J. Chem. Phys. 2011, 135, 054509-054511.

(46) Helbing, J.; Hamm, P. Compact Implementation of Fourier Transform TwoDimensional IR Spectroscopy without Phase Ambiguity. J. Opt. Soc. Am. B 2011, 28, 171-178.

(47) Cringus, D.; Bakulin, A.; Lindner, J.; Pshenichnikov, M. S.; Vohringer, P.; Wiersma, D. A. Ultrafast Energy Transfer in Water-Aot Reverse Micelles. J. Phys. Chem. B 2007, 111, 14193-14207.

(48) Gordon, R. G. Molecular Collisions and Depolarization of Fluorescence in Gases. J. Chem. Phys. 1966, 45, 1643.

(49) Chen, T.; Foley, B.; Ipek, B.; Tyagi, M.; Copley, J.; Brown, C.; Choi, J.; Lee, S.-H. Rotational Dynamics and Its Relation to the Photovoltaic Effect of CH3NH3Pbl3 Perovskite. 2015 preprint arXiv:1506.02205.

(50) Cringus, D.; Jansen, T. L. C.; Pshenichnikov, M. S.; Wiersma, D. A. Ultrafast Anisotropy Dynamics of Water Molecules Dissolved in Acetonitrile. J. Chem. Phys. 2007, 127, 084507.

(51) Bakulin, A. A.; Cringus, D.; Pieniazek, P. A.; Skinner, J. L.; Jansen, T. L.; Pshenichnikov, M. S. Dynamics of Water Confined in Reversed Micelles: Multidimensional Vibrational Spectroscopy Study. J. Phys. Chem. B 2013, 117, 15545-15558.

(52) Woutersen, S.; Bakker, H. J. Resonant Intermolecular Transfer of Vibrational Energy in Liquid Water. Nature 1999, 402, 507-509.

(53) Ji, M.; Gaffney, K. J. Orientational Relaxation Dynamics in Aqueous Ionic Solution: Polarization-Selective Two-Dimensional Infrared Study of Angular Jump-Exchange Dynamics in Aqueous 6m Naclo4. J. Chem. Phys. 2011, 134, 044516.

(54) Quarti, C.; Grancini, G.; Mosconi, E.; Bruno, P.; Ball, J. M.; Lee, M. M.; Snaith, H. J.; Petrozza, A.; Angelis, F. D. The Raman Spectrum of the CH3NH3Pbl3 Hybrid Perovskite: Interplay of Theory and Experiment. J. Phys. Chem. Lett. 2014, 5, 279-284. 